\title{
LISTA RECENZENTÓW „RUCHU PRAWNICZEGO, EKONOMICZNEGO I SOCJOLOGICZNEGO” ZA ROK 2017
}

Jan Barcz

Katarzyna Byrka-Kita

Jan Czempas

Rafał Drozdowski

Maciej Duszczyk

Magdalena Fedorowicz

Tadeusz Gadkowski

Justyna Goździewicz-Biechońska

Joanna Haberko

Piotr Jabkowski

Elżbieta Jantoń-Drozdowska

Leszek Karczewski

Marian Kępiński

Leon Kieres

Marzena Kordela

Barbara Kryk

Grażyna Krzyminiewska

Katarzyna Kuziak

Daniel E. Lach

Irena Lipowicz

Wojciech Łączkowski

Sebastian Majewski

Małgorzata Masternak-Kubiak

Andrzej Matysiak

Danuta Miłaszewicz

Jan Monkiewicz

Tomasz Nieborak

Adam Olejniczak

Monika Oliwa-Ciesielska

Janina Panowicz-Lipska

Jerzy J. Parysek

Witold Płowiec
Krzysztof Podemski

Barbara Pogonowska

Łukasz Pohl

Jakub Pokrzywniak

Bożena Popowska

Anna Przyborowska-Klimczak

Wiesława Przybylska-Kapuścińska

Teresa Rabska

Dorota Rancew-Sikora

Marek Ratajczak

Wanda Ronka-Chmielowiec

Przemysław Rotengruber

Władysław Rozwadowski

Michał Skapski

Małgorzata Słodowa-Hełpa

Marek Smolak

Jerzy Stępień

Hanna Suchocka

Aneta Suchoń

Wanda Sułkowska

Andrzej J. Szwarc

Krzysztof Ślebzak

Paweł Wiliński

Marcin Wiśniewski

Zbigniew Woźniak

Jerzy Zajadło

Robert Zawłocki

Halina Zboroń

Kamil Zeidler

Krystian Ziemski

Maria Zmierczak

Marian Żukowski 